\title{
Indications and contra-indications for minimally invasive mitral valve surgery
}

\author{
Ludwig Müller, Daniel Höfer, Johannes Holfeld, Herbert Hangler, Nikolaos Bonaros, Michael Grimm \\ Department of Cardiac Surgery, Medical University Innsbruck, Innsbruck, Austria \\ Correspondence to: A. Univ. Prof. Dr. Ludwig Müller. Department of Cardiac Surgery, Medical University Innsbruck, Anichstrasse 35, 6020 Innsbruck, \\ Austria. Email: ludwig.mueller@i-med.ac.at.
}

\begin{abstract}
A minimally invasive access through right mini-thoracotomy (MT) for mitral valve surgery (MIMVS) is indicated in all cases except those which cannot be safely addressed by this approach, i.e., in particular major annular calcification (MAC). Other conditions to be regarded as contraindications generally either impose an increased risk of complications compared to the standard approach (e.g., severe aorto-iliac atherosclerosis) or may simply jeopardize the results of MIMVS (any co-morbidity imposing an elevated surgical risk). Partial sternotomy (PS) represents an alternative less invasive approach for cases when MT is not feasible including severe MAC, concomitant aortic valve replacement (AVR) and selected indications for coronary artery bypass graft (CABG). Full sternotomy may be reserved for multiple bypass grafting, use of left internal mammary artery (LIMA), right internal mammary artery (RIMA) and reoperations when a MT approach is not appealing.
\end{abstract}

Keywords: Minimally invasive mitral valve surgery (MIMVS); indications; contraindications

Received: 07 September 2018; Accepted: 13 December 2018; Published: 30 December 2018.

doi: $10.21037 /$ jovs.2018.12.09

View this article at: http://dx.doi.org/10.21037/jovs.2018.12.09

\section{Introduction}

Minimally invasive mitral valve surgery (MIMVS) has not only gained wide spread introduction into clinical practice but also been demonstrated to have certain advantages beyond mere cosmetic benefits in comparison to procedures performed through full sternotomy (1-5). These advantages, however, can only be realized if patient selection is appropriate to guarantee optimal outcomes in regard to repair results, minimal rate of complications, return to normal activity and patient satisfaction in general as well as convincement of referring physicians. To identify factors which might impair results it is necessary to define:

(I) Why certain conditions should be regarded as contraindications;

(II) What the nature of these conditions is;

(III) What might be an alternative less invasive approach to avoid the disadvantages of a full sternotomy; and

(IV) What, if any, are still absolute indications for a full median sternotomy for mitral valve surgery.

MIMVS is not a definitely defined procedure but widely accepted as a procedure using a right mini-thoracotomy (MT), a video-endoscope, with specially designed long shafted instruments and extracorporeal circulation. This is also the definition used for MIMVS in this article (Figure 1). Exact position, shape and length of the incision, usage of a rib retractor, site of vascular access, mode of aortic occlusion-if any-and technique of visualization (totally endoscopic or video assisted direct vision) vary widely among surgeons and there is certainly no "true" minimally invasive procedure although a totally endoscopic procedure without usage of a mechanical rib retractor is mostly regarded as the ultimate goal of trauma reduction in MIMVS. Nevertheless, a partial sternotomy (PS) also has advantages compared to full sternotomy as it is definitely less traumatic and hence less invasive. Its position in the armamentarium of the dedicated mitral valve surgeon is to be addressed in this article. 


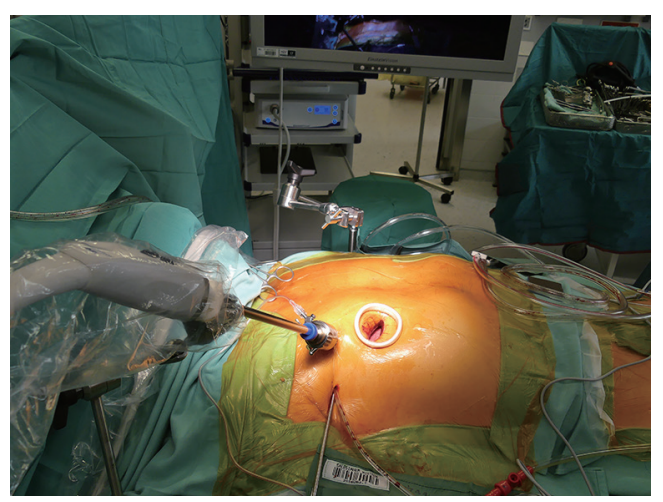

Figure 1 Minimally invasive mitral valve surgery access totally endoscopic approach.

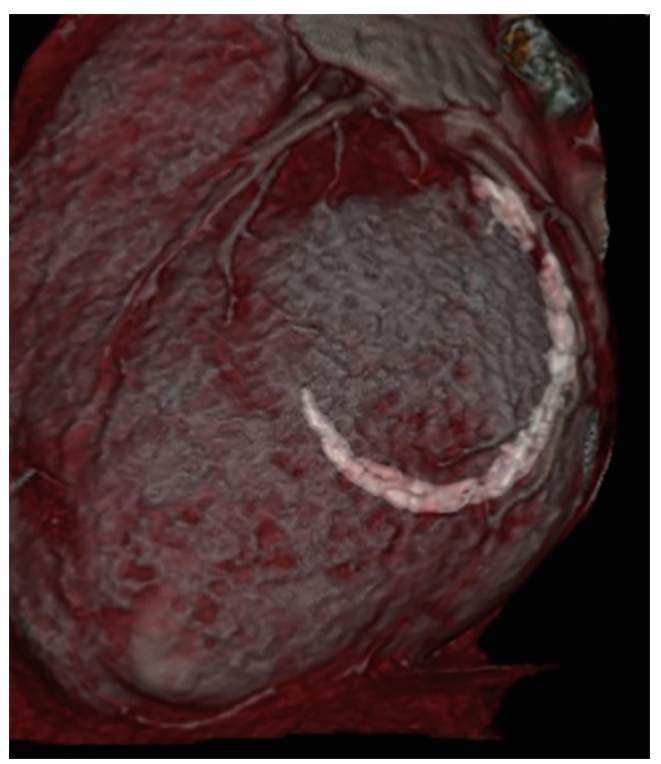

Figure 2 CT scan showing major annular calcification of the posterior mitral annulus.

\section{Indications and contraindications}

\section{Patients selection}

Why are certain conditions regarded as contraindications: anything which increases the risk of complications, risk of a failed repair including long term failure, the likelihood of a bailout conversion to full sternotomy not just offsets the advantages of the minimally invasive approach but may even lead to a worse outcome (6) than a primarily planned larger access. Such conditions are manifold: they may be anatomical or functional.

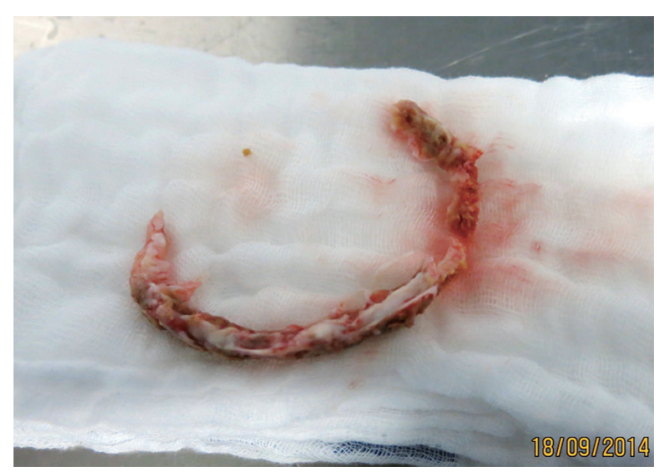

Figure 3 surgical specimen of en bloque resected annular calcification.

\section{Indications}

In general, any isolated or combined mitral valve procedure is amenable for a MT approach except situations when absolute contraindications are identified.

\section{Contraindications}

\section{Valve pathology}

Most conditions apt to valve repair can be treated successfully, especially in degenerative disease. Major annular calcifications (MAC) (Figure 2) cannot be addressed sufficiently especially if en bloque decalcification (Figure 3) of the annulus and annulus repair by sliding atrium plasty and/or pericardial patch reconstruction of the annulus are needed. The angle of the surgical instruments in relation to the mitral valve is very limited and therefore not suitable for complex annulus reconstructions. Advanced rheumatic disease also may be difficult if not impossible to be addressed properly although extended single or bileaflet pericardial patch enlargements can be performed perfectly. Also, infective endocarditis in the active or healed phase can be treated adequately (Figures 4-6) and the rate of successful repairs is comparable to the open approach. Need for valve replacement due to advanced destruction of the valve independent from its etiology is no contraindication by itself but severe calcifications may prohibit the minimal invasive approach due to the inappropriateness of the surgical instruments to deal with major calcifications.

All other valve conditions notably those with severe regurgitation including Barlow's syndrome are excellent cases for a MIMVS repair.

Need for combined surgical procedures especially tricuspid 


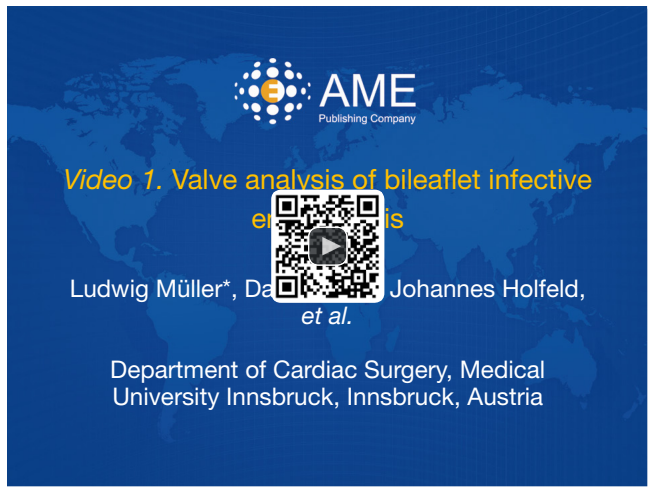

Figure 4 Valve analysis of bileaflet infective endocarditis (7). Available online: http://www.asvide.com/article/view/29183

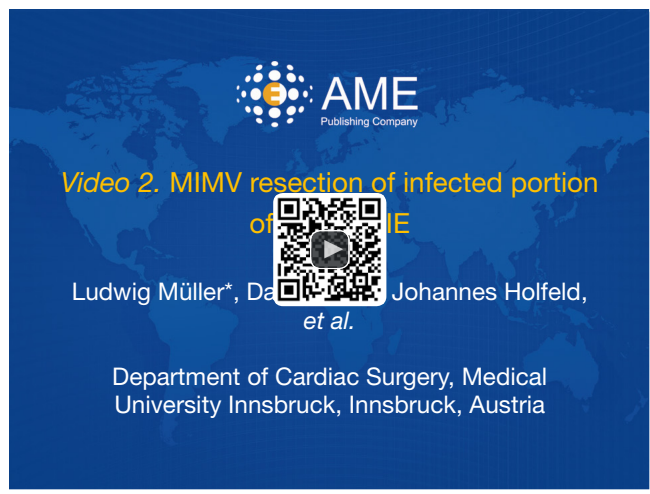

Figure 5 MIMV resection of infected portion of bileaflet IE (8). MIMV, minimally invasive mitral valve; IE, infective endocarditis. Available online: http://www.asvide.com/article/view/29184

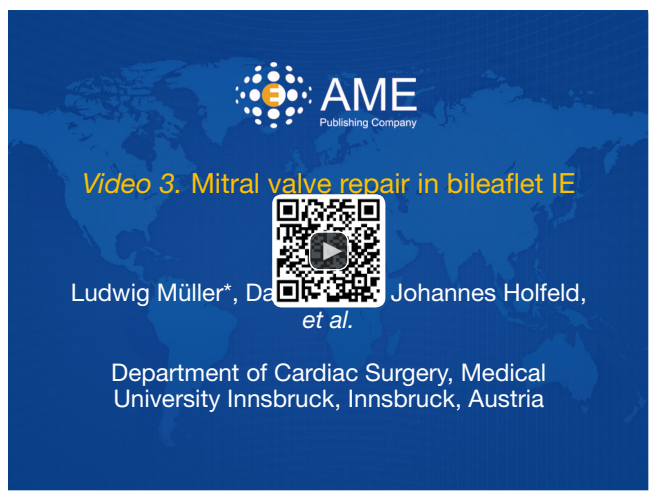

Figure 6 Mitral valve repair in bileaflet IE (9). IE, infective endocarditis. Available online: http://www.asvide.com/article/view/29185

repair, cryo- or radiofrequency ablation of atrial fibrillation and atrial septal defect repair is no contraindication for MIMVS although it must be taken into account that such procedures need additional time. Planning of such combined procedures must consider the expected total ischemic time and in doubt an alternative approach may be preferable.

Concomitant coronary artery bypass grafting $(\mathrm{CABG})$ is a clear contraindication for MIMVS, however, a staged hybrid strategy including percutaneous coronary intervention (PCI) can be reasonable in single or double vessel disease (10).

Simultaneous aortic valve replacement (AVR) has been performed successfully through a MT (11). A larger incision which is located more cranial and medial than in isolated MV surgery and a rib retractor are needed and may be complicated by rib fractures.

\section{Chronic heart failure}

Reduced ejection fraction should not be regarded as contraindication, if the valve procedure is expected to be straight forward and cross clamping time will be short.

\section{Reoperation}

Previous open-heart surgery through median sternotomy or right MT is not a general contraindication. Each case must be evaluated individually and the preferred access can be chosen. Basically, the right MT approach is much less invasive in a re-do situation and preferable especially with patent coronary bypass grafts and after AVR (12). Minimal dissection of the heart is required since the access to the interatrial groove is rather straight. As dissection of the ascending aorta can be impossible from the right side mitral and tricuspid valve surgery can be performed on the beating or fibrillating heart. Ventricular fibrillation is definitely more reliable in prevention of air embolism. Even extensive fibrillation times have been proven safe in respect to myocardial damage if the ventricle is fully vented until the heart resumes its normal action. In combination with continuous $\mathrm{CO}_{2}$ inflation deairing can be achieved safely. If an endoaortic balloon occlusion system is available it can facilitate the procedure markedly since blood leakage through the distorted aortic valve may impair vision considerably and make the valve repair more difficult if the aorta is not occluded. Previous right sided thoracotomy including cardiac procedures are not considered generally as contraindications. Need for prolonged dissection of the lung, however, must be kept in mind and also possible injury to the lungs with prolonged air leakage. If simultaneous signs of lung emphysema are observed extreme caution is advisable.

\section{Aortic conditions}

Definite ascending aortic aneurysm must be treated surgically and hence is a contraindication by itself. Minor dilatation of 


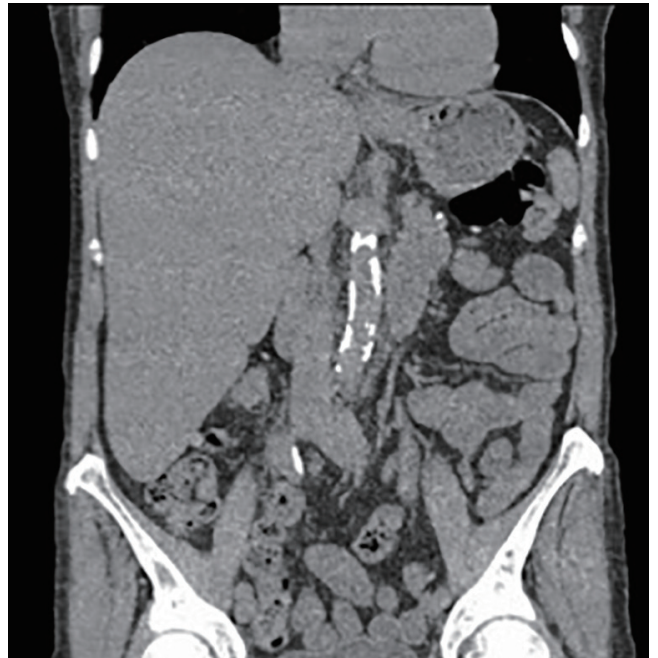

Figure 7 CT scan of calcified abdominal aorta.

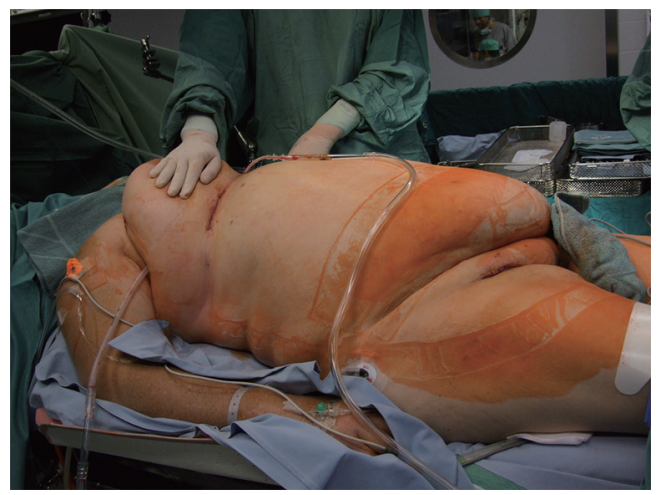

Figure 8 Obese patient successfully operated by MIMVS. MIMVS, minimally invasive mitral valve surgery.

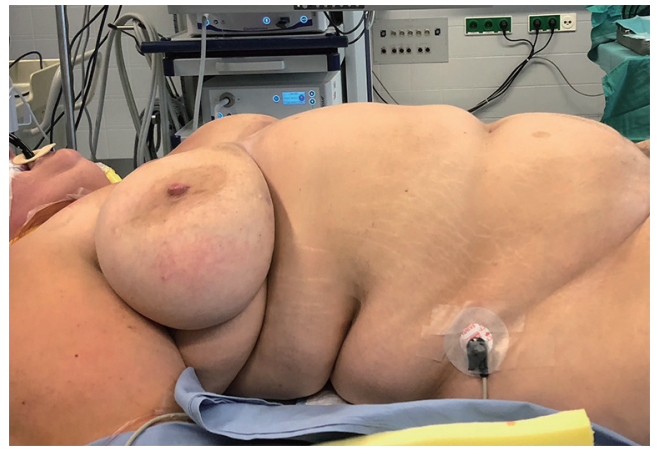

Figure 9 Obese patient inoperable by MIMVS approach. MIMVS, minimally invasive mitral valve surgery. the aorta is no contraindication for MIMS if a transthoracic aortic clamp is used. An endoaortic balloon occlusion is not feasible if the aortic diameter exceeds $40 \mathrm{~mm}$.

Severe calcification of the thoraco-abdominal aorta and iliac-femoral vessels (Figure 7) is a risk factor of cerebral embolism due to retrograde perfusion and has to be regarded as a contraindication for femoral arterial cannulation. Axillary artery cannulation in such cases may be a valid alternative although another less invasive approach may be considered.

\section{General patient factors}

Age

Higher age is not associated with worse outcomes of MIMVS compared to median sternotomy. Due to less trauma and the associated quicker recovery (ventilation and ICU times) it is even preferable to full sternotomy, if no contraindications (e.g., aortic calcifications) are present $(13,14)$.

\section{Obesity}

High BMI is a risk factor for complications following sternotomy and therefore obese patients may benefit even more from MIMVS (Figure 8) than normal BMI patients (15). Mere usage of a soft tissue retractor in such situations often does not provide a sufficient opening in the thoracic wall. A mechanical rib spreading retractor must be used. As long as the branches of the retractor reach the ribs surgery is performed though this tunnel comparable to less obese patients. Excessive obesity especially in combination with very large breasts, however, may impede access to the heart definitely and surgery cannot be performed safely (Figure 9). In such situations full sternotomy may be the only option.

\section{Chest wall deformities and kyphoscoliosis}

A thorough imaging preoperatively and planning of surgery is essential and the right access can be chosen. As in obesity patients with chest deformities may profit from the minimally invasive access even more.

\section{Patient size}

Exceptionally large patients will need additional venous drainage and jugular vein cannulation is necessary. Extra-long instruments have to be on the shelf since the distance from the thoracic wall to the mitral valve and the subvalvular structures is increased.

Unusually small patients and children display other challenges. Due to the small thoracic cavity sufficient space for exposure of the mitral valve may be inappropriate. Femoral vessels in these patients sometimes are too small for direct cannulation. In general patients under $40-45 \mathrm{~kg}$ are rather challenging with a MT approach and alternative approaches should be considered. 


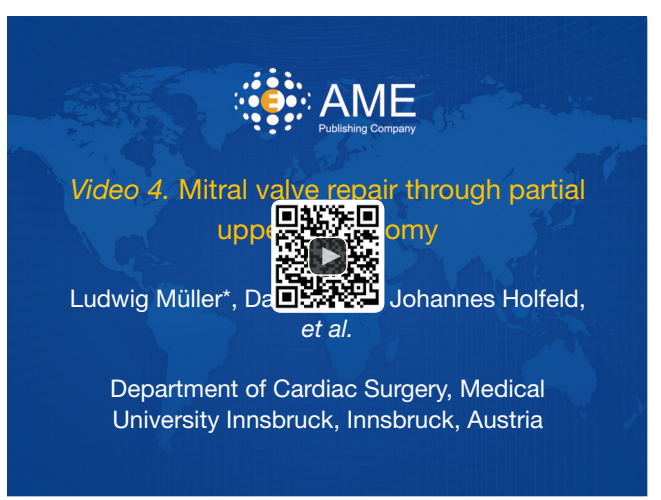

Figure 10 Mitral valve repair through partial upper sternotomy (17). Available online: http://www.asvide.com/article/view/29186

\section{Other conditions}

* Severe lung and/or pleural disease must be judged individually. If severe pleural adhesions are expected dissection may be tedious and a quicker approach can be advantageous. Severe lung emphysema as well as restrictive lung disease impose functional impairment and prolonged ventilator dependence after cardiac surgery and ECC times should be kept as short as possible.

* High pulmonary artery pressure also is a risk factor for postoperative complications and should be considered carefully.

Chronic renal impairment is a risk factor for postoperative renal failure, however, an increased incidence after MIMVS has not been demonstrated.

In general risk factors for perioperative complications will increase the rate of morbidity and mortality and should be observed as contraindications if the results of a MIMVS program should be optimized. On the other hand, especially patients at a higher risk possibly will profit from the reduced trauma of the minimally invasive approach. Finally, individual experience and surgical performance will be key for a successful MIMVS procedure and contraindications depend also on the surgeon's experience. Low numbers of patients suitable for MIMVS in a certain center also should be recognized as contraindication (16).

A wise decision may be the use of an alternative less traumatic approach avoiding full sternotomy but with the advantages of central cannulation, avoidance of a transpleural access and leaving the pericardial sac partially intact.

PS, especially if carried out as an upper hemi-sternotomy is much less traumatic and most if not all mitral valve procedures can be carried out perfectly by the experienced surgeon. No specially designed instruments and no video- endoscopy are needed. Recovery usually is quicker than after full sternotomy, the risk of sternal complications is lower and patients may return to normal activity earlier. And it can be employed in most situations when MT is not appropriate or contraindicated (Figure 10). In reoperative surgery PS is not recommendable since isolation and snaring of the caval veins will be difficult or impossible Coronary bypass grafting to the circumflex artery is not possible in most cases as is the use of a left internal thoracic artery (LITA) graft. Saphenous vein grafts to the right coronary artery (RCA) and-if in certain situations indicated-to the left coronary artery (LAD) can be performed perfectly. Left atrial ablation of atrial fibrillation probably cannot be done properly. All other concomitant procedures like tricuspid repair, interatrial septal procedures and AVR can be done without major difficulties. The most important indications for this approach are severe vascular sclerosis which makes retrograde perfusion too dangerous or even impossible and severe mitral annular calcification requiring either en bloque decalcification or simply stronger instruments to deal with major calcification. And of course, combined aortic and mitral valve surgery can be done with avoidance of full sternal splitting. As the MT approach in most instances is more time consuming also conditions when short bypass and ischemic times are wanted are good indications for hemisternotomy.

At the end, only few hard indications for full sternotomy remain: multiple bypass grafting, use of the internal thoracic arteries, reoperations if not possible through MT and if conversion from MT is necessary.

\section{Identification of contraindications}

According to the nature of conditions which are regarded as contraindications a full preoperative work-up is mandatory: besides the basic echocardiographic investigations coronary artery disease has to be ruled out or diagnosed properly. Left and right heart catheterisation is recommended but may be spared in clear situations. Preoperative TEE is highly recommended but must be performed at least intraoperatively. In addition, all possible candidates for MIMVS should have a contrast medium enhanced CT scan of the body to diagnose and stage aorto-iliac sclerosis and to rule out mitral annular calcifications. In addition, it gives valuable information on the lungs and cardiac anatomy. In some situations, especially when functional MR has to be treated cardiac MRI is helpful. Modern image fusion programs are developing rapidly and adding valuable information. 
In summary severe aorto-iliac atherosclerosis and severe mitral annulus calcification are the most important contraindications for MIMVS, however, other conditions imposing increased risk have to be identified to avoid unnecessary complications and emergency conversion to full sternotomy. Otherwise conditions imposing an elevated risk of functional perioperative complications may bear even less risk when the surgery is performed in a more atraumatic way.

\section{Acknowledgments}

Funding: None.

\section{Footnote}

Provenance and Peer Review: This article was commissioned by the Guest Editor (Peyman Sardari Nia) for the series "Minimally Invasive Mitral Valve Surgery" published in Journal of Visualized Surgery. The article has undergone external peer review.

Conflicts of Interest: All authors have completed the ICMJE uniform disclosure form (available at http://dx.doi. org/10.21037/jovs.2018.12.09). The series "Minimally Invasive Mitral Valve Surgery" was commissioned by the editorial office without any funding or sponsorship. The authors have no other conflicts of interest to declare.

Ethical Statement: The authors are accountable for all aspects of the work in ensuring that questions related to the accuracy or integrity of any part of the work are appropriately investigated and resolved. All procedures performed in studies involving human participants were in accordance with the ethical standards of the institutional and/or national research committee(s) and with the Helsinki Declaration (as revised in 2013). Written informed consent was obtained from the patient for publication of this manuscript and any accompanying images.

Open Access Statement: This is an Open Access article distributed in accordance with the Creative Commons Attribution-NonCommercial-NoDerivs 4.0 International License (CC BY-NC-ND 4.0), which permits the noncommercial replication and distribution of the article with the strict proviso that no changes or edits are made and the original work is properly cited (including links to both the formal publication through the relevant DOI and the license). See: https://creativecommons.org/licenses/by-nc-nd/4.0/.

\section{References}

1. Cheng DC, Martin J, Lal A, et al. Minimally invasive versus conventional open mitral valve surgery: a meta-analysis and systematic review. Innovations (Phila) 2011;6:84-103.

2. Goldstone AB, Atluri P, Szeto WY, et al. Minimally invasive approach provides at least equivalent results for surgical correction of mitral regurgitation: a propensitymatched comparison. J Thorac Cardiovasc Surg 2013;145:748-56.

3. Santana O, Reyna J, Grana R, et al. Outcomes of minimally invasive valve surgery versus standard sternotomy in obese patients undergoing isolated valve surgery. Ann Thorac Surg 2011;91:406-10.

4. Cao C, Gupta S, Chandrakumar D, et al. A meta-analysis of minimally invasive versus conventional mitral valve repair for patients with degenerative mitral disease. Ann Cardiothorac Surg 2013;2:693-703.

5. Downs EA, Johnston LE, LaPar DJ, et al. Minimally Invasive Mitral Valve Surgery Provides Excellent Outcomes Without Increased Cost: A Multi-Institutional Analysis. Ann Thorac Surg 2016;102:14-21.

6. Vollroth M, Seeburger J, Garbade J, et al. Minimally invasive mitral valve surgery is a very safe procedure with very low rates of conversion to full sternotomy. Eur J Cardiothorac Surg 2012;42:e13-5; discusson e16.

7. Müller L, Höfer D, Holfeld J, et al. Valve analysis of bileaflet infective endocarditis. Asvide 2018;5:926. Available online: http://www.asvide.com/article/ view/29183

8. Müller L, Höfer D, Holfeld J, et al. MIMV resection of infected portion of bileaflet IE. Asvide 2018;5:927. Available online: http://www.asvide.com/article/ view/29184

9. Müller L, Höfer D, Holfeld J, et al. Mitral valve repair in bileaflet IE. Asvide 2018;5:928. Available online: http:// www.asvide.com/article/view/29185

10. Santana O, Xydas S, Williams RF, et al. Hybrid approach of percutaneous coronary intervention followed by minimally invasive mitral valve surgery: a 5 -year singlecenter experience. J Thorac Dis 2017;9:S595-601.

11. Lio A, Miceli A, Ferrarini M, et al. Minimally invasive approach for aortic and mitral valve surgery. Eur J Cardiothorac Surg 2016;50:1204-5.

12. Daemen JHT, Heuts S, Olsthoorn JR, et al. Right minithoracotomy versus median sternotomy for reoperative mitral valve surgery: a systematic review and meta-analysis of observational studies. Eur J Cardiothorac 
Surg 2018;54:817-25.

13. Lamelas J, Sarria A, Santana O, et al. Outcomes of minimally invasive valve surgery versus median sternotomy in patients age 75 years or greater. Ann Thorac Surg 2011;91:79-84.

14. Holzhey DM, Shi W, Borger MA, et al. Minimally invasive versus sternotomy approach for mitral valve surgery in patients greater than 70 years old: a propensity-matched comparison. Ann Thorac Surg 2011;91:401-5.

15. Reser D, Sündermann S, Grünenfelder J, et al. Obesity should not deter a surgeon from selecting a minimally

doi: 10.21037/jovs.2018.12.09

Cite this article as: Müller L, Höfer D, Holfeld J, Hangler H, Bonaros N, Grimm M. Indications and contra-indications for minimally invasive mitral valve surgery. J Vis Surg 2018;4:255. invasive approach for mitral valve surgery. Innovations (Phila) 2013;8:225-9.

16. Holzhey DM, Seeburger J, Misfeld M, et al. Learning minimally invasive mitral valve surgery: a cumulative sum sequential probability analysis of 3895 operations from a single high-volume center. Circulation 2013;128:483-91.

17. Müller L, Höfer D, Holfeld J, et al. Mitral valve repair through partial upper sternotomy. Asvide 2018;5:929. Available online: http://www.asvide.com/article/ view/29186 